\title{
CONTRIBUIÇÃO AO CONHECIMENTO DAS ESPÉCIES DE CYPERACEAE DA ÁREA DE PROTEÇÃO AMBIENTAL MUNICIPAL DO INHAMUM, CAXIAS, MARANHÃO
}

1.Daniele Souza dos Santos; 2.Eduardo Oliveira Silva; 3.Gonçalo Mendes da Conceição

${ }^{1,2,3}$ Núcleo de Pesquisa dos Recursos Biológicos dos

Cerrados Maranhenses/RBCEM,Laboratório de Biologia Vegetal/LABIVE,

Centro de Estudos Superiores de Caxias/Universidade Estadual do Maranhão

CESC/UEMA, Morro do Alecrim,CEP 65604-380, Caxias/MA, Brasil

*Autor para correspondência - hyophila@yahoo.com.br

Recebido em: 03/01/2014 - Aprovado em: 04/04/2014 - Publicado em: 12/04/2014

\section{RESUMO}

O estudo foi realizado com o objetivo de listar e atualizar as espécies da família Cyperaceae, ocorrentes no município de Caxias/MA. Para isso, foram realizadas várias coletas de material botânico na Área de Proteção Ambiental Municipal do Inhamum. Como resultado, listou-se 19 espécies, com adição de mais cinco (Cyperus haspan, C. iria, C. surinamensis, Diplacrum capitatum e Eleocharis geniculata) ao número de espécies conhecidas no município.

PALAVRAS-CHAVE: Cerrado; Cyperus; Flora do Maranhão

\section{CONTRIBUTION TO THE KNOWLEDGE OF SPECIES OF CYPERACEAE IN THE ENVIRONMENTAL PROTECTION AREA OF INHAMUM, CAXIAS, MARANHÃO, BRAZIL}

\begin{abstract}
The aim of this study is to survey and update the Cyperaceae species from city of Caxias, Maranhão, Brazil. Expeditions were done in the Environmental Protection Area of Inhamum for collection of botanical material, area of cerrado in the municipality. Was confirmed 19 species, five of them new occurrences in the municipality.
\end{abstract}

KEYWORDS: Cerrado, Cyperus; Flora from Maranhão

\section{INTRODUÇÃO}

A família Cyperaceae compreende ervas de pequeno a grande porte, cespitosas, perenes, com folhas laminares a lineares, reunidas na base de ramos aéreos ou distribuídas ao longo destes, de escapo triangular, caule do tipo rizomatoso, inflorescência em espigueta e frutos do tipo aquênio (RIBEIRO et al., 1999; SOUZA \& LORENZI, 2008; JUDD et al., 2009).

Possui uma abrangência cosmopolita, ocupando preferencialmente áreas de ecossistemas úmidos a alagados, sazonal ou permanentemente, assim como ambientes florestais (GOETGHEBEUR, 1998; OLIVEIRA, 2011), embora alguns 
gêneros como Bulbostylis Kunth apresentem maior diversidade em áreas de cerrado e campos secos (PRATA et al., 2007; SILVEIRA \& LONGHI-WAGNER (2008).

Em relação a sistemática de Cyperaceae, esta foi incluída por CRONQUIST (1988), junto à Poaceae na ordem Cyperales. Porém, estudos filogenéticos com dados moleculares, revelaram uma maior afinidade de Cyperaceae com a família Juncaceae, estando posicionada na ordem Poales (CHASE, 2003; APG III, 2009).

No Brasil, ocorrem 628 espécies de Cyperaceae, distribuídas em 42 gêneros, onde Rhynchospora Vahl e Cyperus L. são os mais representativos em números de espécies, sendo listadas no estado do Maranhão, 43 espécies em 19 gêneros (ALVES et al., 2014). Com o objetivo de aumentar o conhecimento sobre as espécies de Cyperaceae ocorrentes no estado do Maranhão, este estudo traz um inventário florístico dessas espécies na Área de Proteção Ambiental Municipal do Inhamum, no município de Caxias/MA.

\section{MATERIAL E MÉTODOS}

\section{ÁREA DE ESTUDO}

A área de Proteção Ambiental Municipal do Inhamum (045ㅜ30"S e 4324'53'W), localiza-se à margem esquerda da BR-316, sendo cortada verticalmente pela MA-127 que liga o município de Caxias/MA a São João do Sóter/MA, possuindo uma área de aproximadamente 4.500 ha. Esta área está inserida no Município de Caxias, situado na Mesorregião do Leste Maranhense, com área de 5.223,98 $\mathrm{Km}^{2}$ (SOUZA \& CONCEICÃO, 2009). Caracteriza-se por apresentar uma vegetação típica de cerrado, que vai desde Campo limpo até Cerradão, entremeadas por babaçuais. Ao longo dos córregos, lagoas e nascentes, a vegetação é formada por Mata Ciliar ou de Galeria com árvores de grande porte. A área é banhada pelo Rio Itapecuru, e junto com seus afluentes, formam diversos banhos naturais, fazendo, portanto, parte da sua bacia hidrográfica (SOUZA \& CONCEICÃO, 2009; CONCEICÃO et al., 2010).

Foram realizadas excursões mensais nas diferentes regiões fitofisiográficas da área de estudo para coleta do material botânico no período entre 2012 a 2013. Estes foram coletados e herborizados com base nas técnicas usuais em taxonomia de MORI et al., (1989) e FIDALGO \& BONONI (1989), identificados por especialista e incorporados à coleção do Herbário Prof. Aluízio Bittencourt/HABIT, do Centro de Estudos Superiores de Caxias, da Universidade Estadual do Maranhão CESC/UEMA.

\section{RESULTADOS E DISCUSSÃO}

Foram encontrados 19 táxons de Cyperaceae na Área de Proteção Ambiental Municipal do Inhamum, distribuídos em sete gêneros (Cyperus, Diplacrum R. Brown, Eleocharis R. Brown, Fimbristylis Vahl, Fuirena Rottb., Kyllinga Rottb. e Rhynchospora).

Entre os gêneros listados neste estudo, Cyperus foi considerado o mais importante, não somente pelo número de espécies coletadas (10 espécies), mas pela abundância de indivíduos observados em campo, e sua ocorrência em diferentes ambientes. A riqueza de Cyperus o torna o segundo maior gênero dentro de Cyperaceae, embora, Carex L. é o gênero com maior número de espécies, seguindo após estes, Fimbristylis, Rhynchospora, entre outros (GOETGHEBEUR, 1998). Nesse levantamento, Kyllinga e Fimbristylis apresentaram duas espécies cada, e os demais gêneros com apenas uma única espécie (Quadro 1). 
QUADRO 1: Lista de Espécies e Gêneros encontrados na Área de Proteção Ambiental Municipal do Inhamum, Caxias/Maranhão.

\begin{tabular}{l|l}
\hline \multicolumn{1}{c|}{ Gêneros } & \multicolumn{1}{c}{ Espécies } \\
Cyperus & $\begin{array}{l}\text { Cyperus aggregatus (Willd.) Endl, C. eragrostis Lam, C. haspan L, C. } \\
\text { iria L, C. laxus Lam, C. luzulae (L.) Retz, C. surinamensis Rottb, C. } \\
\text { liguralis L, C. virens Michx, C. distans (L.). }\end{array}$ \\
\hline Kyllinga & Kyllinga vaginata Lam, K. brevifolia Rottb, K. odorata Vahl. \\
\hline Fimbristylis & Fimbristylis capillaris A. Gray, F. dichotoma (L.) Vahl. \\
\hline Rhynchospora & Rhynchospora corymbosa (L.) Britton. \\
\hline Eleocharis & Eleocharis geniculata (L.) Roem \& Schult. \\
\hline Diplacrum & Diplacrum capitatum (Willd.) Boeck. \\
\hline Fuirena & Fuirena umbellata Rottb. \\
\hline
\end{tabular}

A primeira lista florística de espécies de Cyperaceae para o município de Caxias/MA, foi elaborada por SOUZA \& CONCEIÇÃO (2009), que catalogaram 16 espécies, distribuídas em sete gêneros. Posteriormente, NUNES et al. (2012), registraram 15 espécies para Área de Proteção Ambiental Municipal do Inhamum, sendo que o presente estudo atualiza essa ultima listagem florística para a área de estudo em mais cinco espécies (Cyperus haspan, C. iria, C. surinamensis, Diplacrum capitatum e Eleocharis geniculata).

\section{CONCLUSÃO}

O acréscimo do número de espécies de Cyperaceae obtido nesse trabalho revela que mais esforços de coleta são necessários para a área de estudo, já que trabalhos taxonômicos em áreas de proteção tornam-se importantes ferramentas para ampliação do conhecimento botânico-ecológico nessas unidades de conservação.

\section{AGRADECIMENTOS}

À Universidade Estadual do Maranhão/UEMA, pela concessão da Bolsa de Iniciação Científica do primeiro autor. Ao Centro de Estudos Superiores de 
Caxias/CESC, por disponibilizar toda a infraestrutura de laboratório e de campo para a realização da pesquisa.

\section{REFERÊNCIAS}

ALVES, M.; HEFLER, S. M.; TREVISAN, R.; LUZ, C. L. Cyperaceae. in: Lista de Espécies da Flora do Brasil. Rio de Janeiro: Jardim Botânico do Rio de Janeiro. 2014. Disponível em: <http://floradobrasil.jbrj.gov.br/jabot/floradobrasil/FB100>. Acesso em: 9 Mar. 2014.

APG. III (Angiosperm Phylogeny Group). An update of the Angiosperm Phylogeny Group Classification for the orders and families of flowering plants: APG III. Botanical Journal of the Linnean Society, v. 161, n. 2, p. 105-121, 2009.

CHASE, M. An update of the Angiosperm Phylogeny Group classification for the orders and families of flowering plants: APG II. Botanical Journal of Linnean Society, v. 141, n. 4, p. 399-436. 2003.

CONCEICÃO, G. M.; RUGGIERI, A. C.; GUIMARÃES, E. R. Melastomataceae da Área de Proteção Ambiental Municipal do Inhamum, Caxias, Maranhão. Revista de Biologia e Farmácia, v. 4, n. 2, p. 83-88, 2010.

CRONQUIST, A. The evolution and classification of flowering plants. 2 ed. New York: The New York Botanical Garden. 1988. 555p.

FIDALGO, O.; BONONI, V. L. R. Técnicas de coleta, preservação e herborização de material botânico. São Paulo: Instituto de Botânica. 1989. 62 p.

GOETGHEBEUR, P. Cyperaceae. In: KUBITZKI, K. (Ed.). The families and genera of vascular plants. Monocotyledons. Hamburg: Springer. v. 4, 1998. p. 141-190.

JUDD, W. S.; CAMPBELL, C. S.; KELLOG, E. A.; STEVENS, P. F.; DONOGHUE, M. J. Sistemática Vegetal: um Enfoque Filogenético, 3. Ed. Porto Alegre: Artmed. 2009. $612 \mathrm{p}$.

MORI, S. A.; SILVA, L. A. M.; LISBOA, G.; CORADIN, L. Manual de Manejo de Herbário Fanerogâmico. $2^{a}$ ed. Ilhéus, Centro de Pesquisas do Cacau - CEPLAC. Porto Alegre, RS, 1989. 104 p.

NUNES, C. S.; CONCEIÇÃO, G. M.; SILVA, E. O. Levantamento das Espécies de Cyperaceae Juss. na Área de Proteção Ambiental Municipal do Inhamum, Caxias, Maranhão, Brasil. Enciclopédia Biosfera, Centro Cientifico Conhecer, Goiânia, v. 8, n. 15, p. 1796-1800, 2012.

OLIVEIRA, A. L. R.; Gil. A. S. G.; BOVE, C. P.; Hydrophytic Cyperaceae from the Araguaia river basian, Brasil. Rodriguésia v. 4, n. 62, p. 847-866, 2011.

PRATA, A. P.; MENEZES, N. L.; MAZZONI-VIVEIROS, S. C.; WANDERLEY, M. G.; THOMAS, W. W. Anatomia do escapo e rizoma de espécies brasileiras de Bulbostylis Kunth (Cyperaceae). Revista Brasileira de Botânica, v. 30, n. 2, p. 245256, 2007. 
RIBEIRO, J. E. L. S.; HOPKINS, M. J. G.; VICENTINI, A.; SOTHERS, C. A.; COSTA, M. A. S.; BRITO, J. M.; SOUZA, M. A. D.; MARTINS, L. H. P.; LOHMANN, L. G.; ASSUNÇÃO, P. A. C. L.; PEREIRA, E. C.; SILVA, C. F.; MESQUITA, M. R.; PROCÓPIO, L. C. Flora da Reserva Ducke: Guia de identificação das plantas vasculares de uma floresta de terra firme na Amazônia Central. Manaus, INPA, 1999. $816 \mathrm{p}$.

SILVEIRA, G. H.; LONGHI-WAGNER, H. M. Cyperaceae Juss. no Morro Santana Porto Alegre e Viamão, Rio Grande do Sul, Brasil. Iheringia, Sér. Bot., v. 63, n. 2, p. 295-320, 2008.

SOUZA, C. E. O.; CONCEICÃO, G. M. Espécies de Cyperaceae de ocorrência no município de Caxias, Maranhão, Brasil. Pesquisa em Foco, v. 17, n. 2, p. 26-31, 2009.

SOUZA, V. C.; LORENZI, H. Botânica sistemática: guia ilustrado para identificação das famílias de fanerógamas nativas e exóticas no Brasil, baseado em APG II. 2. ed. Nova Odessa, SP: Instituto Plantarum, 2008. 704p. 